\title{
siRNA targeting the IRF2 transcription factor inhibits leukaemic cell growth
}

\author{
${\text { AILYN } \text { CHOO }^{1} \text {, PATRICIA PALLADINETTI }}^{1}$, TIFFANY HOLMES ${ }^{2}$, SHREERUPA BASU $^{2}$, SYLVIE SHEN $^{1}$, \\ RICHARD B. LOCK ${ }^{1}$, TRACEY A. O'BRIEN ${ }^{2}$, GEOFF SYMONDS ${ }^{1}$ and ALLA DOLNIKOV ${ }^{1,2}$ \\ ${ }^{1}$ Children's Cancer Institute Australia for Medical Research; ${ }^{2}$ Sydney Cord \& Marrow Transplant Facility, \\ Sydney Children's Hospital, High Street, Randwick NSW 2031, Australia
}

Received January 11, 2008; Accepted February 27, 2008

\begin{abstract}
Interferon regulatory factor (IRF) 1 and its functional antagonist IRF2 were originally discovered as transcription factors that regulate the interferon- $\beta$ gene. Control of cell growth has led to the definition of IRF 1 as a tumour suppressor gene and $I R F 2$ as an oncogene. Clinically, approximately $70 \%$ of cases of acute myeloid leukaemia demonstrate dysregulated expression of $I R F 1$ and/or $I R F 2$. Our previous studies have shown that human leukaemic TF-1 cells exhibit abnormally high expression of both $I R F 1$ and $I R F 2$, the latter acting to abrogate IRF1 tumour suppression, making these cells ideal for analysis of down-regulation of IRF2 expression. A novel G418 screening protocol was developed and used for identifying effective siRNA that targets IRF2 (siIRF2). Using optimized siIRF2 in leukaemic TF-1 cells, IRF2 was downregulated by approximately $70 \%$ at both mRNA and protein levels. Phenotypically, this resulted in growth inhibition associated with $\mathrm{G}_{2} / \mathrm{M}$ arrest as well as induction of polyploidy, differentiation and apoptosis. In contrast to these results, siIRF2 targeting did not affect normal haematopoietic stem/progenitor cell growth. These results indicate the potential utility of IRF2 inhibition as a therapeutic approach to cancer.
\end{abstract}

\section{Introduction}

Acute myeloid leukaemia (AML) can be viewed as a clonal myeloproliferative disorder of the haematopoietic stem/progenitor cell (HSC/HPC) characterised by the rapid expansion of differentiation-arrested cells of varying maturity, often caused by distinct molecular mechanisms (1). These molecular mechanisms include leukaemia-associated fusion proteins and mutations that function to aberrantly activate either signalling

Correspondence to: Dr Alla Dolnikov, Sydney Cord \& Marrow Transplant Facility, High Street, Randwick NSW 2031, Australia E-mail: alla.dolnikov@sesiahs.health.nsw.gov.au

Key words: interferon regulatory factor 1 and 2, leukaemogenesis mechanisms or transcriptional regulators thereby directly interfering with haematopoietic cell growth and differentiation (1).

Mutations that create constitutively active Ras proteins are among the most frequently detected genetic alterations in human leukaemia (2), occurring in approximately $30 \%$ of AML cases (3). While frequently activated in haematopoietic malignancies, the manner in which Ras activation contributes to human leukaemia is not well understood. The Ras protein has a crucial role in many haematopoietic regulatory processes and has been the target of many therapeutic approaches $(3,4)$. Mutant $N$-ras $\left(N\right.$-ras $\left.{ }^{m}\right)$ is known to impair murine myelopoiesis and erythropoiesis both in ex vivo tissue culture models and in vivo in bone marrow reconstituted mice where it confers susceptibility to haematopoietic disorders that resemble human AML (4). In addition, $N$-ras ${ }^{m}$ was shown to induce myeloproliferation when transduced into human primitive HSC/HPC both ex vivo in long-term culture and in vivo in a 'humanized' NOD/SCID mouse model (5).

Paradoxically, ectopic expression of $\mathrm{N}-$ ras $^{m}$ was shown to induce tumour suppressive pathways in some leukaemic cell lines, namely K562 and U937 but not in TF-1 cells (6). High-throughput microarray expression analysis revealed up-regulation of the transcription factor and tumour suppressor, interferon regulatory factor (IRF) 1 when $\mathrm{N}$-ras ${ }^{m}$ was constitutively expressed in leukaemic K562 and U937 cells; but not in TF- 1 cells that carries a mutation in the endogenous N-ras gene and exhibited high basal levels of IRF1 (6). Increased levels of IRF1 in K562 and U937 cells induced up-regulation of the tumour suppressor gene, $p 21^{\text {WAFI }}$ and as expected, antisense-mediated IRF1 suppression resulted in reduced levels of $p 21^{\text {WAFI }}$ (6). Results indicated that this activation cascade is responsible for the tumour suppressor effects observed in K562 and U937 cells (6). Up-regulation of IRF1 via ectopic expression of $N$-ras ${ }^{m}$ may provide a potential tumour suppressive approach for leukaemia therapy (6). The IRF1 gene is located on chromosome 5q31.1, an area commonly deleted in myelodysplasia, AML and acute promyelocytic leukaemia (APL) resulting in inactivation of one or both alleles (7-9).

Surprisingly, in a recent study, IRF1 was found to be highly expressed in a significant proportion of primary AML specimens (10). In addition, high IRF 1 expression was seen 
in human leukaemia TF-1 cells (6). We hypothesized that IRF1 tumour suppressor activity was abrogated in these AMLs by secondary mutations (11). Retroviral cDNA library analysis was performed to screen for the genes that abrogate $N$-ras ${ }^{m}$-induced growth arrest (11). This analysis identified IRF2 as an abrogating factor in the growth arrest pathway (11). IRF2 over-expression in U937 cells abrogated $\mathrm{N}$-ras ${ }^{m_{-}}$ induced growth suppression through the repression of $p 21^{\text {WAFI }}$ (11). In addition, high IRF2 expression was found in leukaemia TF-1 cells (6). Overall, these results established an $\mathrm{N}$-ras ${ }^{m}$ mediated growth suppression pathway whereby the balance between IRF1 and IRF2 expression (IRF1:IRF2 ratio) appears to be the determining factor.

We report here that down-regulation of $I R F 2$ by specific siRNA in TF-1 cells increases the IRF1:IRF2 ratio allowing IRF1 and p21 ${ }^{\mathrm{WAF} 1}$-related tumour suppressor effects. These effects included growth suppression, cell cycle arrest and concomitant polyploidisation. In addition, targeting IRF 2 in leukaemic TF-1 cells resulted in a reduced fraction of clonogenic leukaemic blasts. By contrast, IRF2 down-regulation in normal cord blood $\mathrm{CD} 34^{+}$primitive progenitor cells did not affect cell growth. To the best of our knowledge, IRF2 targeting is a novel concept that may have therapeutic potential.

\section{Materials and methods}

Cell culture. TF-1 cells were derived from the myeloid progenitor cell population recovered from a patient with erythroleukaemia (American Type Culture Collection (ATCC), Manassas, VA). U937 cells (ATCC) were derived from a patient's diffuse histiocytic lymphoma. Both cell types were cultured in Roswell Park Memorial Institute (RPMI)-1640 (Gibco BRL) medium containing $10 \%$ fetal calf serum (FCS), $2 \mathrm{mM}$ L-glutamine, $50 \mathrm{U} / \mathrm{ml}$ penicillin, $50 \mu \mathrm{g} / \mathrm{ml}$ streptomycin, $25 \mathrm{mM}$ HEPES buffer (RPMI medium) and incubated at $5 \% \mathrm{CO}_{2}$ and $37^{\circ} \mathrm{C}$. Recombinant interleukin-3 (IL-3) (10 ng/ml) was also added for TF-1 cells. U937-derived IRF2/Neo or Neo-only cells were produced as previously described (11). G418 antibiotic selection of these cells was performed at $1000 \mu \mathrm{g} / \mathrm{ml}$. Umbilical cord blood (UCB) was obtained from healthy full-term deliveries through the Australian Cord Blood Bank, with Human Ethics approval and donor's informed consent. UCB CD34+ cells were purified using the MACS CD34 isolation kit from Miltenyi Biotec (Auburn, CA). Purified CD34+ cells were prestimulated for $48 \mathrm{~h}$ in Iscove's Modified Dulbecco's Medium (IMDM) (SigmaAldrich, St. Louis, MO) with 10\% FCS, 2 mM L-glutamine, $100 \mathrm{U} / \mathrm{ml}$ penicillin $/ 100 \mu \mathrm{g} / \mathrm{ml}$ streptomycin, and a cytokine cocktail containing FMS-like tyrosine kinase ligand (FLT3L), stem cell factor (SCF), thrombopoietin (TPO); and interleukin-6 (IL-6); all at $100 \mathrm{ng} / \mathrm{ml}$ (R\&D Systems, Minneapolis, $\mathrm{MN})$.

siRNA transfection. siRNA oligonucleotides were synthesized and annealed for use (Qiagen Inc., Valencia, CA). For analysis, $5 \times 10^{5}$ cells per well were seeded onto a 24-well plate. Cells were transfected with $1.5 \mu \mathrm{g}(280 \mathrm{nM})$ siRNA; either scrambled non-silencing siRNA (AAUUCUCCGAACGU GUCACGUTT) or siRNA targeting IRF2 (siIRF2, CUCUUU AGAAACUGGGCAATT) (12) using the RNAiFect Trans- fection Kit Protocol (Qiagen Inc.). All experiments were performed in triplicate and compared to cells incubated in lipofection reagent alone (untreated). For electroporation experiments, $1 \times 10^{7} \mathrm{TF}-1$ cells were resuspended in $500 \mu 1$ serum-free RPMI media containing $3 \mu \mathrm{M}$ siRNA. Cells were electroporated at $250 \mathrm{~V}, 500 \mathrm{uF}$. siRNA uptake was analysed in the FITC-labelled scrambled oligonucleotide control at $6 \mathrm{~h}$ for both lipofection and electroporation. siRNA uptake was analysed by flow cytometry.

Flow cytometry. Flow cytometry analyses were performed using a FACSCalibur instrument and CellQuest analysis software (Becton Dickinson, San Jose, CA). Events (1x104) were collected in each experiment. The FL1 channel was used to measure FITC-labelled siRNA and siRNA uptake was measured in live cells; gating based on forward (FSC) and side scatter (SSC) characteristics. The percentage of FITClabelled cells and mean fluorescent intensity (MFI) were analysed in each sample. For phenotyping, cells $\left(1 \times 10^{6}\right)$ were blocked with mouse $\mathrm{IgG}$ and stained with monoclonal antibodies against CD45, CD34, CD33, CD14 and CXCR4 (Becton Dickinson). Events $\left(1 \times 10^{5}\right)$ were acquired and data were analysed with CellQuest (Becton Dickinson). CD34 ${ }^{+}$ percentages were calculated as percentages of the lymphocyte gate (set on $\mathrm{CD}_{4} 5^{+}$, low side scatter cells of the unexpanded UCB), excluding CD14+ cells. Propidium iodide (PI) was included to assess UCB viability.

Clonogenic assays. Clonogenic assays were performed in Methylcellulose (StemCell Technologies Inc., Vancouver, $\mathrm{BC}$, Canada). TF- 1 cells $\left(5 \times 10^{2}\right)$ were cultured in $1 \mathrm{ml}$ methylcellulose in a 12-well plate for 8 days and single colony counts were scored. Samples were then washed in PBS and counted for statistical analysis. UCB CD $34^{+}$cells were plated in Methocult GF H4534 [1\% methylcellulose in IMDM, 30\% FBS, $1 \%$ BSA, $10^{-4} \mathrm{M}$ 2-mercaptoethanol, $2 \mathrm{mM}$ L-glutamine, $50 \mathrm{ng} / \mathrm{ml} \mathrm{SCF}, 10 \mathrm{ng} / \mathrm{ml}$ granulocyte-macrophage colonystimulating factor (GM-CSF), $10 \mathrm{ng} / \mathrm{ml} \mathrm{IL-3} \mathrm{(StemCell}$ Technologies)] supplemented with $3 \mathrm{U} / \mathrm{ml}$ of erythropoietin (EPO) and $50 \mathrm{ng} / \mathrm{ml}$ granulocyte-growth stimulating factor (G-CSF) (R\&D Systems) as previously described (5). Cells $\left(1 \times 10^{3}\right)$ suspended in $1.1 \mathrm{ml}$ were plated in each $35-\mathrm{mm}$ dish. Colony forming units (CFU) were counted on day 14 and classified into three categories: pure erythroid (CFU-E), granulomonocytic (CFU-GM), and total (CFU).

Cell cycle analysis. Cells $\left(1 \times 10^{5}\right)$ were harvested, permeabilized and stained using $50 \mu \mathrm{g} / \mathrm{ml}$ PI, 10\% Triton X-100, $1 \mathrm{M}$ sodium citrate in phosphate buffered saline (PBS). Samples were incubated at room temperature for $45 \mathrm{~min}$ and then analysed by flow cytometry, measuring cell stages based on DNA content: $\mathrm{G}_{1}$-cells $(2 \mathrm{~N})$, S-cells $(2 \mathrm{~N}-4 \mathrm{~N})$ and $\mathrm{G}_{2} /$ M-cells (4N) where $\mathrm{N}$ is haploid content. Apoptotic cells were identified as cells with sub $\mathrm{G}_{1}(<2 \mathrm{~N})$ content.

Morphological analysis. Cells $\left(1 \times 10^{4}\right)$ were suspended in $100 \mu \mathrm{PBS}$, fixed onto slides using a Cytospin centrifuge (800 rpm for $10 \mathrm{~min}$ ) and then stained with May and Grunwald and Giemsa stains and analysed by light microscopy. Cells with more than two nuclei were identified as polyploid. Mitotic 
cells at different stages of cell cycle were identified by their condensed chromosomes. Polyploid and mitotic indexes were measured in 10 fields, 100 cells per field; 1000 cells were quantified.

Semi-quantitative reverse transcriptase polymerase chain reaction ( $R T-P C R)$. Total RNA was isolated according to the manufacturer's instructions using TRIzol reagent (Invitrogen Corp., Carlsbad, CA). Total RNA ( $1 \mu \mathrm{g})$ was transcribed using the Reverse Transcription Kit (Promega Corporation, Madison, WI) and an iCycler (Bio-Rad, Hercules, CA) at $25^{\circ} \mathrm{C}$ for $10 \mathrm{~min}, 45^{\circ} \mathrm{C}$ for $1 \mathrm{~h}$. Amplification was performed using Amplitaq Gold (Applied Biosystems, Foster City, CA) according to standard protocols. B-actin conditions were: denaturation at $94^{\circ} \mathrm{C}$ for $10 \mathrm{~min}$ followed by 20-30 cycles of $94^{\circ} \mathrm{C}$ for $30 \mathrm{sec}, 55^{\circ} \mathrm{C}$ for $30 \mathrm{sec}, 72^{\circ} \mathrm{C}$ for $30 \mathrm{sec}$. An additional elongation period of $72^{\circ} \mathrm{C}$ for $5 \mathrm{~min}$ was performed before storage at $4^{\circ} \mathrm{C}$. Reverse-transcribed IRF2 RNA was amplified within the linear range. PCR conditions for IRF2 cDNA amplification were $94^{\circ} \mathrm{C}$ for $30 \mathrm{sec}, 58^{\circ} \mathrm{C}$ for $30 \mathrm{sec}$ and $72^{\circ} \mathrm{C}$ for $45 \mathrm{sec}$. Reaction mixtures were amplified for 30 cycles followed by an elongation period. Products were separated on a $1 \%$ agarose gel, visualized by ethidium bromide staining and quantified using a VersaDoc Imaging System (Bio-Rad) and normalized to B-actin.

Real-time RT-PCR. Total RNA was isolated using TRIzol reagent. Samples were reverse transcribed to cDNA using the Reverse Transcription Kit (Promega). Samples were divided into four equal aliquots, mixed with Taqman Mastermix (Applied Biosystems) and specific or $\beta_{2}$-microglobulin Taqman probe (Applied Biosystems). Gene expression analysis was performed using an ABI Prism 7700 Sequence Detection System (Applied Biosystems).

Western blot analysis. Total cellular protein (48 $\mu \mathrm{g})$ was run on a pre-cast $4-15 \%$ gradient polyacrylamide gel (Bio-Rad). Samples were then transferred onto an Hybond-C nitrocellulose membrane (Amersham Biosciences, Piscataway, NJ) and stained with Ponceau $\mathrm{S}$ for visualization of protein bands. Samples were blocked with 5\% non-fat dairy milk (NFDM) and probed for $B$-actin, IRF2 (sc-13042- Santa Cruz Biotechnology Inc., Santa Cruz, CA), IRF1 (612046-BD) and p21 $1^{\text {WAF1 }}$ (OP64-Calbiochem, San Diego, CA) followed by anti-mouse and anti-rabbit conjugated horseradish peroxidase secondary antibodies (Amersham Biosciences) and visualized by chemiluminescence (Pierce Biotechnology Inc., Rockford, IL). Quantification and detection was performed by X-ray film and a VersaDoc Imaging System (Bio-Rad).

Statistical analysis. The Student's t-test was used to evaluate significance in each set of values. Results are presented as p-values where $<0.05$ was considered significant.

\section{Results}

Testing siIRF2 function using G418 selection. The U937 cell line was chosen for this analysis because these cells exhibit naturally low levels of IRF2 $(6,13)$. U937 cells were transduced with a Moloney murine leukaemia virus (MoMLV) retroviral vector, containing IRF2 and the neomycin phosphotransferase $\left(\mathrm{Neo}^{\mathrm{R}}\right.$ ) gene to produce IRF2/Neo cells (Fig. 1A). We hypothesized that targeting IRF2 by siRNA in IRF2/Neo cells would act to reduce mRNA and protein expression for both IRF2 and neomycin phosphotransferase proteins and result in increased sensitivity to G418. A Neo-only vector lacking the IRF2 gene was used to produce control Neo-only cells. Transfection efficiency of siRNA in U937 was found to be $79 \%$ (Fig. 1B). When IRF2/Neo cells were transfected with IRF2 siRNA and then cultured in the presence of G418, a marked decrease in cell growth and viability was observed, while Neo-only cells were not affected (Fig. 1C). Cell cycle analysis showed a marked accumulation of cells in $\mathrm{G}_{2} / \mathrm{M}$ $48 \mathrm{~h}$ post-transfection in IRF2/Neo- but not Neo-only cells; siIRF2-treated IRF2/Neo-cells displayed partial-recovery at $72 \mathrm{~h}$ and full-recovery at $120 \mathrm{~h}$ post-treatment (Fig. 1E). IRF2/Neo-cells treated with scrambled siRNA showed no change in cell cycle profile but some growth reduction compared to Neo-only cells (Fig. 1E). Control Neo-only cells showed no change in cell growth following silRF2 or scrambled siRNA transfection (Fig. 1B). siIRF2 caused a marked reduction of IRF2 protein levels in IRF2/Neo cells but not in cells treated with scrambled oligonucleotides, in fact some up-regulation of IRF2 protein was seen in these cells (Fig. 1D). These results demonstrated the utility of this test system for silRF2 function and specificity.

IRF2 down-regulation in leukaemic TF-1 cells. We next examined the effect of IRF2 down-regulation in TF-1 cells previously shown to express high IRF2 and IRF1 compared to the human leukaemic cell lines, K562 and U937 (6). In addition, we have demonstrated that enforced expression of IRF2 acts to protect K562 and U937 cells from tumour suppressor activity of IRF1 (11). Here we hypothesized that high IRF2 acts to abrogate the tumour suppressor activity of IRF1 in TF-1 cells (11). Thus, down-regulation of IRF2 in TF-1 cells should re-activate IRF1-mediated growth suppression.

An siRNA uptake of $92 \%$ by lipofection (Fig. 2D, top panel) and $94 \%$ by electroporation (Fig. 2D, bottom panel) was observed in TF-1 cells. Lipofection induced a greater relative MFI ( $\sim 20$ compared to 3$)$ indicating a greater siRNA uptake per cell. While there was no impact on cell viability of samples after lipofection, by contrast a high loss of cell viability $(\sim 60 \%)$ was observed after electroporation. For these reasons (greater uptake per cell and no impact on cell viability), lipofection was routinely used.

Untreated cells showed increasing IRF2 mRNA expression with time, while siIRF2 showed a marked suppression of gene expression, as determined by mRNA levels; $60 \%$ suppression was observed $72 \mathrm{~h}$ post-transfection (Fig. 2A). Seventy percent of IRF2 protein suppression was observed $48 \mathrm{~h}$ post-treatment (Fig. 2B). To determine specificity of effect, IRF1 protein levels were also quantified. IRF1 has high sequence homology with IRF2. Results showed a steady increase in IRF1 protein levels over time in both untreated and siIRF2 treated cells (Fig. 2C). When measured, a transient ( $24 \mathrm{~h}$ post-transfection) 6.5-fold increase of $p 21^{\text {WAFI }}$ transcript relative to untreated cells was seen in TF-1 cells transfected with siIRF2 (Fig. 2E). Up-regulation of $p 21^{W A F I}$ was consistent with the growth 
A

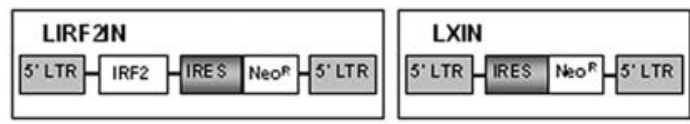

C
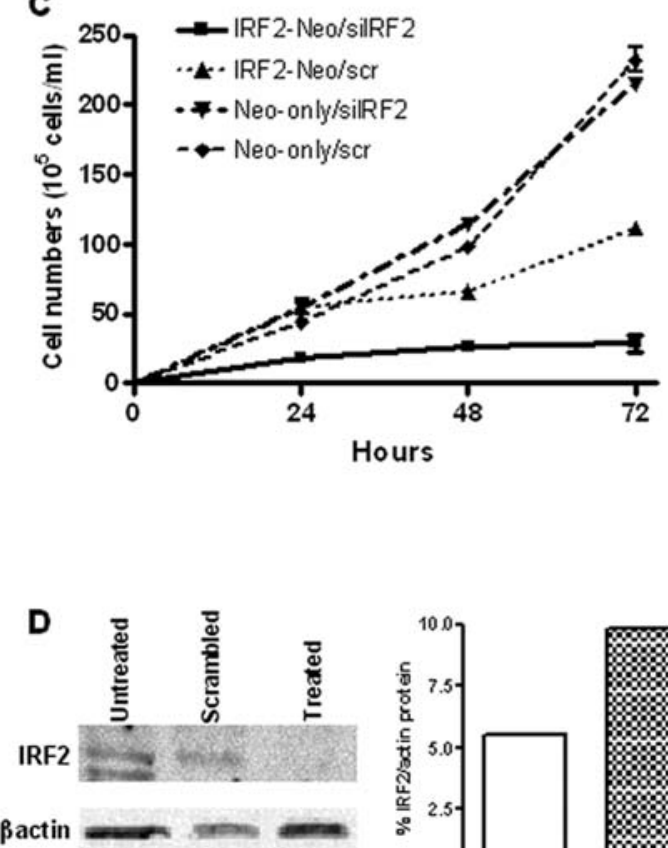

$\mathbf{B}$
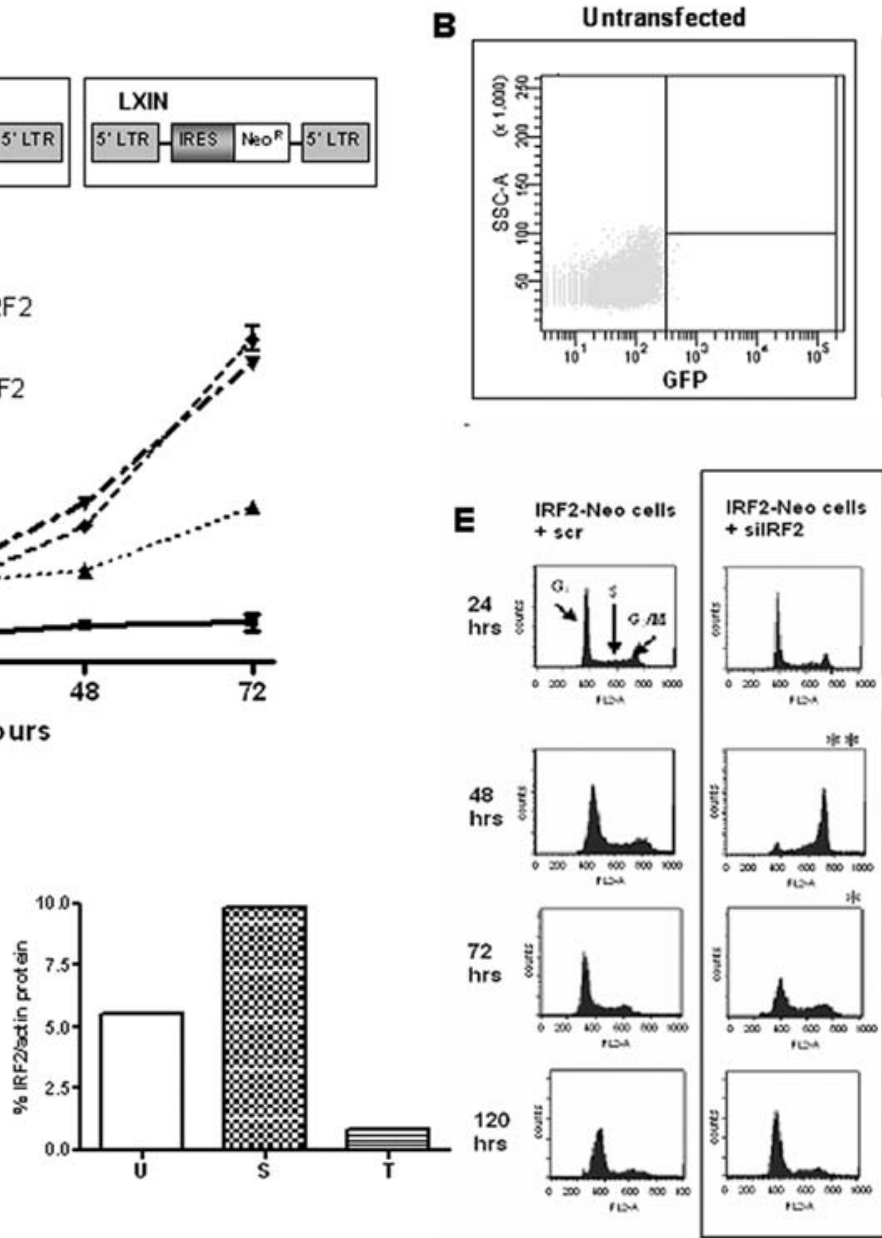

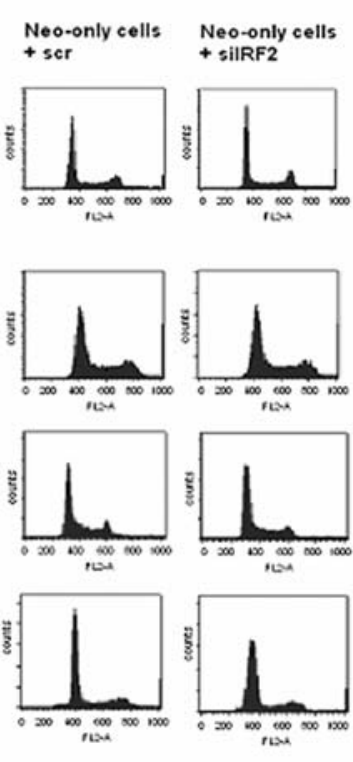

Figure 1. siIRF2 effects cell growth and cell cycle in test cells. (A) Schematic depiction of vector constructs used for U937 siRNA experiments. U937 cells naturally have a low IRF2 basal level. Exogenous material is introduced into these cells to upregulate IRF2 protein. This Moloney murine leukaemia virus based vector (LIRF2IN) is designed with an internal ribosome entry site (IRES) allowing independent translation of both the IRF2 gene and the neomycin phosphotransferase (NeoR) which offers a means of vector plasmid selection. The vector is also flanked by long terminal repeats (LTR) (left panel). Another similar construct (LXIN) lacking the IRF2 gene was designed as a control for siIRF2 functional specificity (right panel). (B) U937 uptake of FITC-labelledsiRNA analysed by fluorescent microscopy. (C) Down-regulation of IRF2 by siRNA inhibits growth of IRF2/Neo cells but not Neo-only cells in the presence of G418. $\mathrm{p}<0.05$, when comparing IRF2-Neo cells treated with siIRF2 to IRF2-Neo cells treated with scrambled oligonucleotides. (D) Western blot analysis of IRF2 protein showed marked IRF2 protein suppression in IRF2-Neo cells $48 \mathrm{~h}$ post siRNA treatment. U, untreated cells; S, cells transfected with scrambled oligonucleotides; T, cells treated with siIRF2. (E) Cell cycle analysis of U937 cells post siIRF2 treatment showed $\mathrm{G}_{2} / \mathrm{M}$ arrest in IRF2-Neo cells treated with siIRF2 at $48 \mathrm{~h}$, followed by partial recovery at $72 \mathrm{~h}$ and full recovery at $120 \mathrm{~h}$. All other samples remained unaffected. Similar results were obtained from 3 independent experiments; a representative experiment is shown.

suppression observed in TF-1 cell cultures. siIRF2 caused a marked growth inhibition at 48 and $72 \mathrm{~h}$ post-treatment (Fig. 3A). TF-1 cells treated with scrambled siRNA showed some reduction in cell proliferation at the $72 \mathrm{~h}$ time-point (Fig. 3A) presumably due to a non-specific IFN-response (14). Clonogenic assays were performed to examine the surviving fraction of leukaemic cells at 24 and $48 \mathrm{~h}$ post-treatment. A clear reduction in both colony numbers (Fig. 3B, left panel) and the cell numbers (Fig. 3B, right panel) within the colonies was observed in siIRF2-treated samples in comparison to controls. An $\sim 2$-fold reduction in colony numbers and a 4-fold reduction in cell numbers was seen (Fig. 3B).

Cell cycle analysis showed a marked accumulation of TF-1 cells in $\mathrm{S}_{-} \mathrm{G}_{2} / \mathrm{M} 48 \mathrm{~h}$ post-transfection (Fig. 3C, bottom left panel). siIRF2-treated cells displayed full recovery in cell cycle profile at $72 \mathrm{~h}$ post-treatment (Fig. 3C, bottom right panel). By contrast, TF-1 cells treated with scrambled siRNA showed no significant changes in cell cycle (data not shown).

Morphological analysis showed a marked increase in mitotic (Fig. 3D, left panel) and polyploid cell (Fig. 3D, right panel) indexes in suspension culture at 48 and $72 \mathrm{~h}$ posttransfection. Thus, the reduction in the number of clonogenic blasts observed in clonogenic assay correlated with the $G_{2} / M$ growth arrest (Fig. 3C, bottom left panel) and formation of nondividing polyploid cells observed following siIRF2 treatment.

Leukaemic TF-1 cells express the primitive haematopoietic progenitor cell marker CD34 and are arrested at an early differentiation stage (6). We analysed whether siIRF2 treatment acted to induce differentiation of TF- 1 cells. Following transfection with siIRF2, cell samples were tested for CD14 (myeloid cell marker) and CD34 (primitive haematopoietic progenitor cell marker) expression by flow cytometry. A 
A

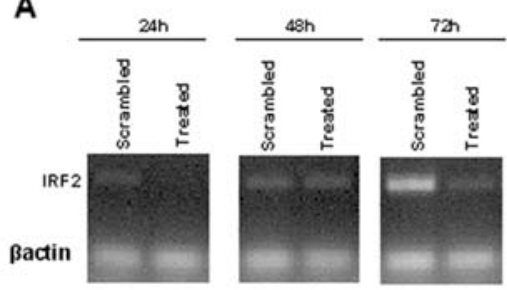

B
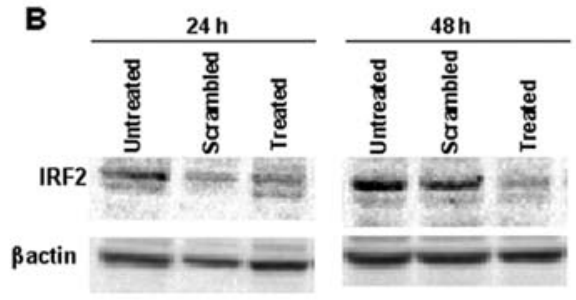

C

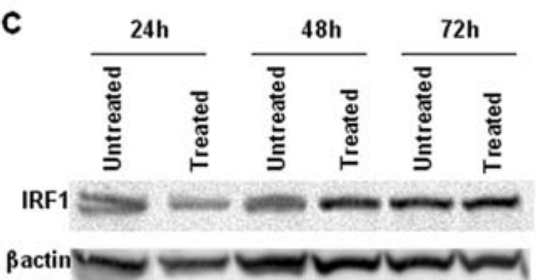

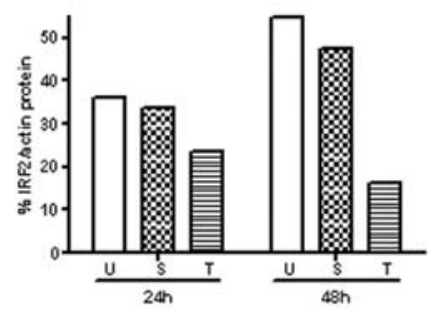

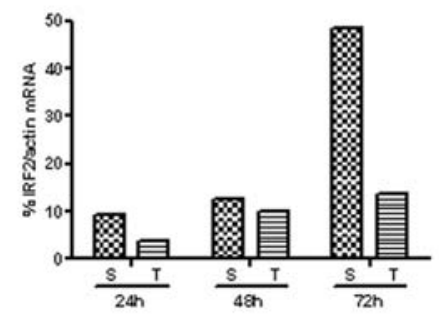

D
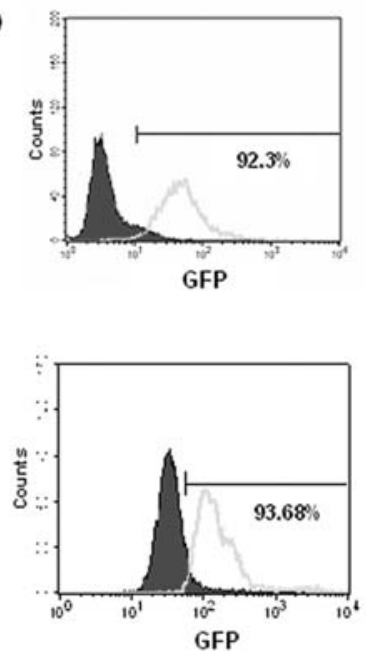

E

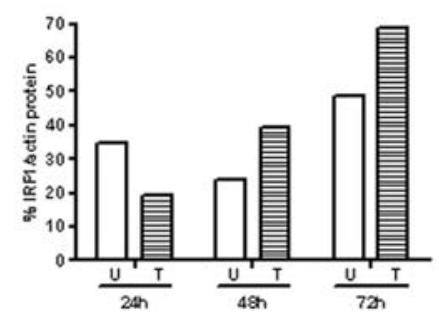

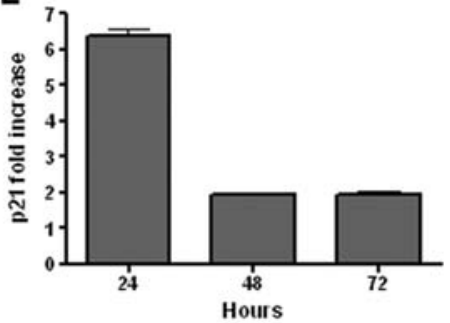

Figure 2. Expression of IRF1, IRF2 and $p 21^{\text {WAFI }}$ in TF-1 cells in response to siIRF2. (A) RT PCR results showed IRF2 mRNA suppression at 72 h post treatment. (B) Western blot analysis showed IRF2 protein suppression at $48 \mathrm{~h}$ post treatment. (C) Western blot analysis showed a steady increase in IRF1 protein expression up to $72 \mathrm{~h}$ post treatment. (D) FACS analysis of TF-1 cells transfected with FITC labelled scrambled (non-silencing) siRNA. siRNA uptake was $92 \%$ via lipofection while uptake by electroporation was 94\%. (E) Real-time RT PCR results showed $p 21^{\text {WAFI }}$ upregulation $24 \mathrm{~h}$ post treatment. Similar results were obtained in 3 independent transfection experiments; in A, B and C, results of one representative experiment are shown. U, untreated cells; S-cells treated with scrambled oligonucleotides; $\mathrm{T}$, cells treated with siIRF2.

marked decrease of CD34 expression was observed in siIRF2treated culture (relative mean fluorescence intensity, MFI of 10.7 compared to 28.9 in cells treated with scrambled oligonucleotides and 21 in untreated cells) (Fig. 4A), while CD14 expression remained relatively constant (data not shown). Flow cytometry revealed a shift in IgG staining in the siIRF2-treated sample apparently due to the increased autofluorescence characteristic of monocytic differentiation (Fig. 4A). In addition, a marked shift in forward-scatter (FSC) (Fig. 4B) and an increased proportion of cells with high side scatter (SSC) was seen in siIRF2-treated cells (12\%) compared to that observed $(6 \%)$ in cells treated with scrambled siRNA. An increase in FSC indicates cell enlargement characteristic of monocytic differentiation. An increase in SSC indicates increased cell granularity, also known to become more intense in differentiating myeloid cells. As a positive control, phorbol-12-myristate-13-acetate (PMA), a known inducer of myeloid differentiation (15), was used and, as expected, was found to reduce CD34 expression and increase SSC/FSC parameters while not modifying CD14 expression in TF-1 cells (data not shown). However, in contrast to PMA-treated cells, siIRF2-treated TF-1 cells did not exhibit increased adherence to the plastic, and no morphological features of advanced myeloid differentiation were observed.

The increase in mitotic and polyploid cells observed in siIRF2 treated TF-1 cells, and the unstable nature of such polyploid cells led us to expect higher cell death. To examine this, cells were transfected with siIRF2 and at $72 \mathrm{~h}$ FCS and IL-3 were withdrawn as an inducer of cell death. Cell numbers were determined every $24 \mathrm{~h}$ both before and after factor withdrawal. Results showed a more significant reduction in cell viability in treated cells compared to untreated and scrambled samples (siIRF2 treated samples showed a 13\% and $11 \%$ reduction in viability compared to untreated and scrambled samples respectively, $\mathrm{p}=0.0414$ ) (Fig. 4C). Overall, these results show that siIRF2 treatment inhibits leukaemic cell growth in TF-1 cells by suppression of cell cycling, formation of polyploid cells, induction of myeloid differentiation and reduced viability, all of which act to reduce the numbers of clonogenic blasts in the treated culture.

Down-regulation of IRF2 does not affect normal haematopoiesis. UCB-derived CD34+ cells were transfected with $1.5 \mu \mathrm{g}$ siRNA by lipofection; the identical treatment to that used for TF-1 cells. We examined whether the pre-stimulation with cytokines that promote $\mathrm{CD} 34^{+}$cell cycling is needed for efficient siRNA uptake. UCB CD $34^{+}$cells pre-stimulated with cytokines exhibited $90 \%$ uptake of FITC-labelled oligonucleotides similarly to unstimulated cells, however, a greater relative MFI ( $\sim 300$ compared to 120 when normalised to the autofluorescence shown by the grey line, Fig. 5A) was found compared to cells that were not pre-stimulated with the 

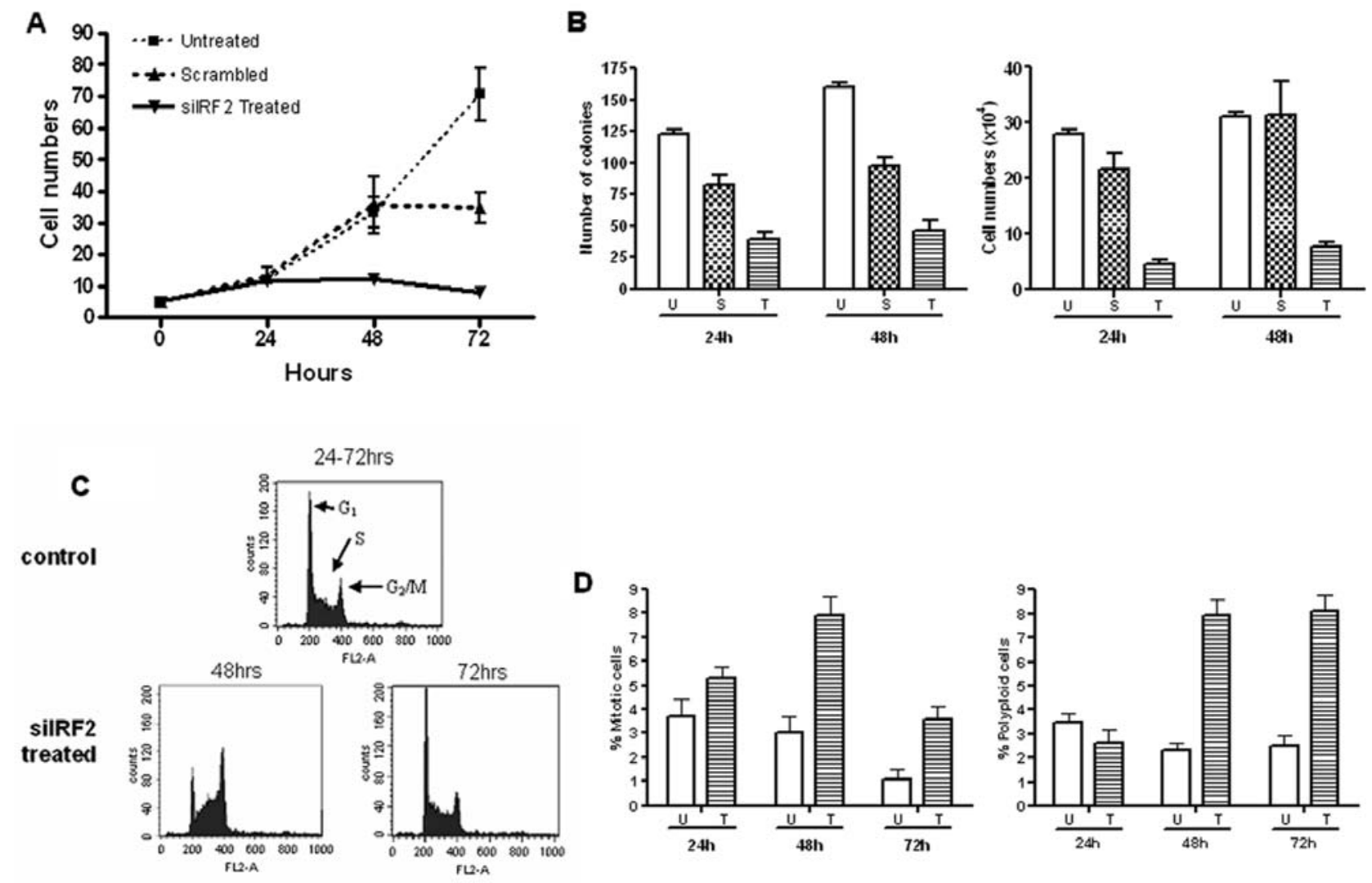

Figure 3. siIRF2 inhibits TF-1 cell growth. (A) siIRF2 treatment inhibits TF-1 cell growth in suspension culture. p<0.05, when comparing siIRF2-treated cells to the cells treated with scrambled oligonucleotides or untreated cells at 48 and $72 \mathrm{~h}$ time-points. (B) Clonogenic assay performed at 24 and $48 \mathrm{~h}$ after siIRF 2 transfection showed a decreased number of colonies (left panel) and reduction in cell numbers in the treated samples (right panel). (C) Cell cycle analysis of TF-1 cells treated with siIRF2. $\mathrm{G}_{2} / \mathrm{M}$ arrest was induced in TF-1 cells $48 \mathrm{~h}$ following siIRF2 treatment: siIRF2-treated cells exhibit shift in PE-fluorescence from $\mathrm{G}_{1}$ (top panel) to $\mathrm{S}-\mathrm{G}_{2} / \mathrm{M}$ populations (bottom left panel), $48 \mathrm{~h}$ following siRNA transfection. (D) silRF2-treated cells demonstrated increased percentage of mitotic cells (left panel) and polyploid cells (right panel) at 48 and $72 \mathrm{~h}$ time-points post-treatment. U, untreated cells; S, cells treated with scrambled oligonucleotides; T, cells treated with siIRF2.

cytokines, indicating a greater siRNA uptake per cell (Fig. 5A). Fluorescent microscopy also demonstrated efficient uptake of the labelled oligonucleotides in both un-prestimulated
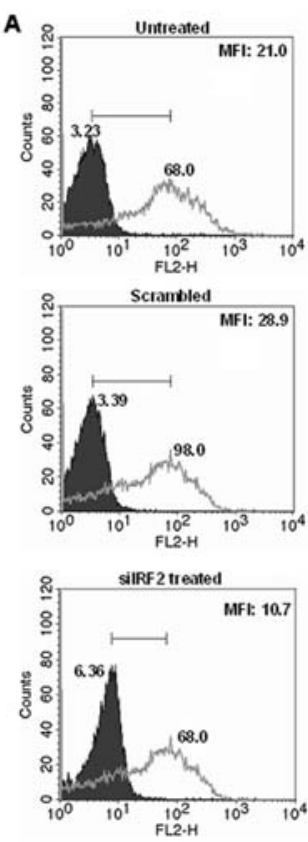
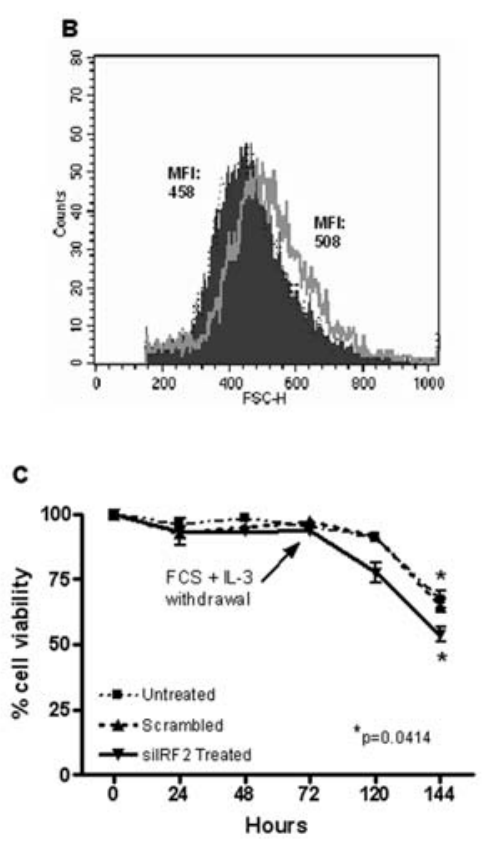

(Fig. 5A, left panel) and pre-stimulated cells (Fig. 5A, right panel). It is relevant that pre-stimulation with cytokines resulted in the significant expansion of total cell numbers (2-fold) and induction of myeloid differentiation with $40 \%$ of cells not expressing CD34 anymore (5). The uptake per cell was similar in $\mathrm{CD}_{34}{ }^{+}$and CD34- cells (Fig. 5C). These results showed that siRNA uptake can occur with low cell cycling of CD34 ${ }^{+}$ cells (no pre-stimulation) but is increased by pre-stimulation with cytokines most probably due to increased cell cycling.

When pre-stimulated $\mathrm{CD} 34^{+}$cells were transfected with siIRF2, $80 \%$ gene suppression was observed (Fig. 5D).

Figure 4. siIRF2 promotes some features of differentiation in TF-1 cells. (A) Flow cytometry analysis of the CD34 antigen expression in untreated (top panel), scrambled siRNA-treated (middle panel) and siIRF2-treated (bottom panel) TF-1 cells. Solid line-IgG control, grey line-CD34-specific fluorescence. A significant shift in IgG fluorescence is apparently due to the increased autofluorescence seen in siIRF2-treated cells (bottom panel). MFI is indicated for IgG- and CD34-specific fluorescence. Relative MFI (MFI for CD34 divided by MFI for IgG) is shown in the top left corner of each panel. (B) An increased forward-scatter cells (FSC) in siIRF2-treated cells (grey line) compared to control (solid line) was observed at 48 h. (C) siIRF2treated samples showed a 13 and $11 \%$ reduction in viability compared to untreated and scrambled samples respectively, $\mathrm{p}=0.0414$. 

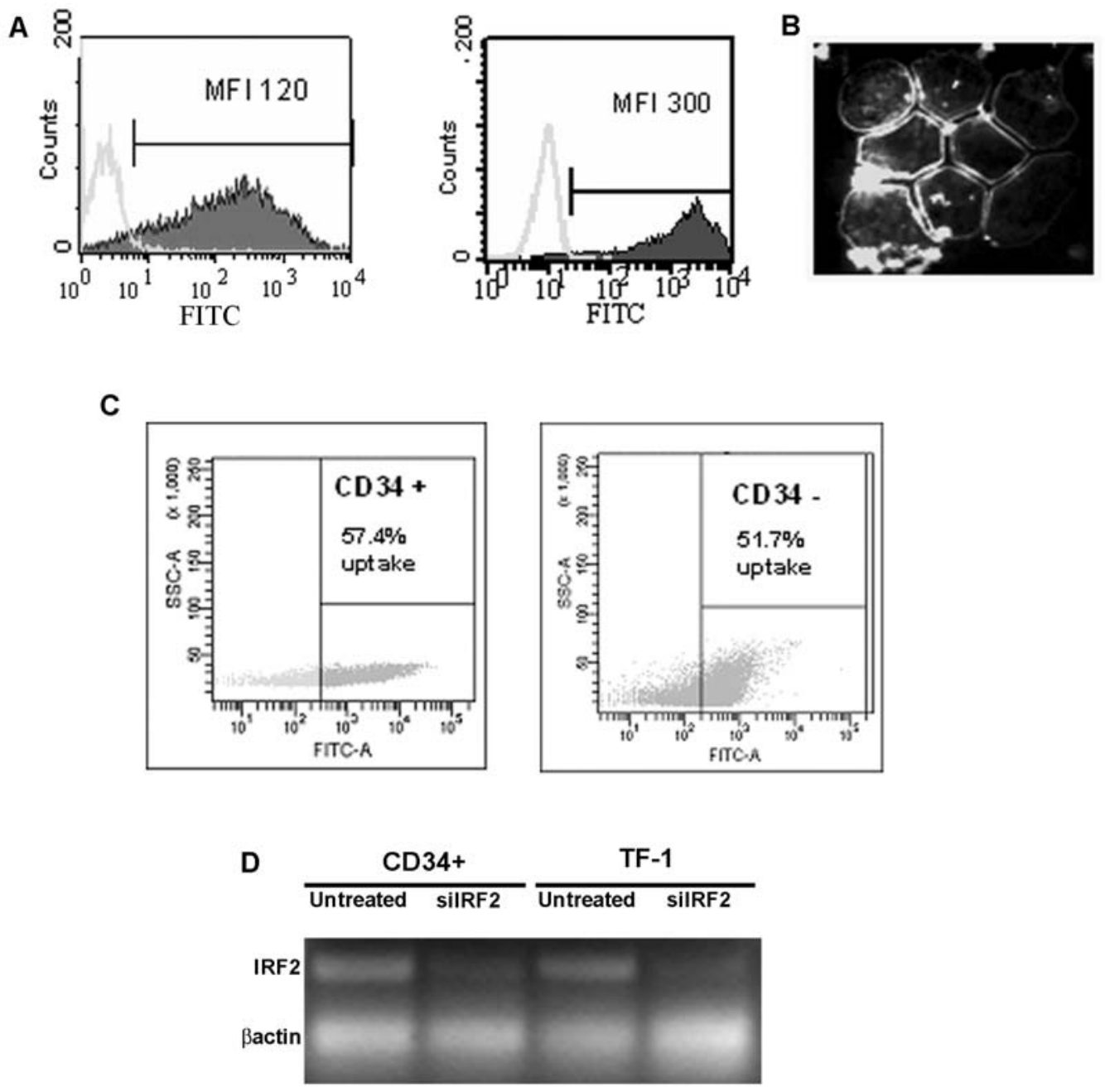

Figure 5. siIRF2 uptake and modulation of IRF2 expression in normal haematopoietic CD34+ cells. (A) Pre-stimulation with cytokines increases uptake of siRNA in primary cord blood-derived CD $34^{+}$cells. CD $34^{+}$cells were transfected with FITC-labelled siRNA without pre-stimulation with cytokines (left panel) or pre-stimulated with cytokines $48 \mathrm{~h}$ prior transfection with siRNA (right panel). Solid line represents fluorescence produced by cells transfected with FITClabelled siRNA and lipofection reagent; grey line, fluorescence produced by the cells treated with lipofection reagent alone. (B) siRNA uptake of FITClabelled-siRNA analysed by fluorescent microscopy. (C) Uptake of siRNA by CD34+ and CD34+ was analysed by flow cytometry. No difference was observed. (D) RT PCR analysis of IRF2 mRNA suppression in CD34+ at $48 \mathrm{~h}$ post transfection. Gene suppression (80\%) was observed in primary CD34 ${ }^{+}$cells. This is similar to the level of IRF2 down-regulation seen in leukaemic TF-1 cells (92\%).

This is similar to the level of IRF2 down-regulation seen in leukaemic TF-1 cells (92\%) (Fig. 5D). Notably, the basal level of IRF2 mRNA expression appears to be similar in $\mathrm{CD} 34^{+}$cells and TF- 1 cells (Fig. 5D). However, in contrast to TF-1 cells, the growth and viability of siIRF2-transfected CD34+ cells was not affected by the treatment (Fig. 6A). Flow cytometry analysis demonstrated that the expression of CD34 was not affected by silRF2 treatment (Fig. 6B). Some reduction (from 57 to $46 \%$ ) in the proportion of $\mathrm{CD}^{2} 4^{+}$cells, though, was observed in cells treated with scrambled siRNA (Fig. 6B), as determined by flow cytometry. When analysed in clonogenic assays, total colony numbers as well as the numbers of CFU-E (erythroid) and CFU-GM (myeloid) colonies were found to be not affected by siIRF2 treatment (Table I). It is relevant that cells transfected with the scrambled oligonucleotides exhibited some inhibition of clonogenic activity that was consistent with the inhibition of cell growth in suspension culture (Table I). Overall, these results show that IRF2 suppression does not inhibit growth of normal haematopoietic progenitor cells.
Table I. Clonogenic growth of cord blood CD34-positive cells following siIRF2 transfection.

\begin{tabular}{lrrr}
\hline & CFU-total & CFU-GM & CFU-E \\
\hline Control & $226 \pm 0.0$ & $218.5 \pm 1.5$ & $7.5 \pm 1.5$ \\
siIRF2 treated & $214.5 \pm 5.5$ & $208.5 \pm 3.5$ & $6 \pm 2.0$ \\
Scrambled & $187 \pm 6.0$ & $181.5 \pm 7.5$ & $5.5 \pm 1.5$
\end{tabular}

$\mathrm{CD}_{3} 4^{+}$cells were transfected with siIRF2 or scrambled oligonucleotides. Transfected cells were analysed in clonogenic assay $48 \mathrm{~h}$ following transfection. Untreated cells were used as additional control. $10^{3}$ cells per $\mathrm{ml}$ were plated in $1 \%$ methylcellulose supplemented with cytokines (Materials and methods). For each assay, cells were plated in duplicate. Granulomonocytic colony forming unit (CFU-GM), erythroid colony forming unit (CFU-E), and total colony number (CFU-total) were scored on day 14 . Mean \pm SEM values for colony numbers are presented. 


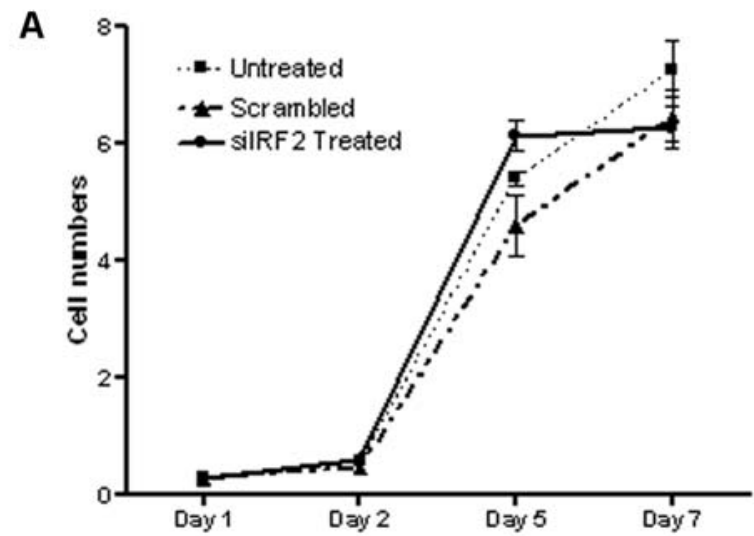

B

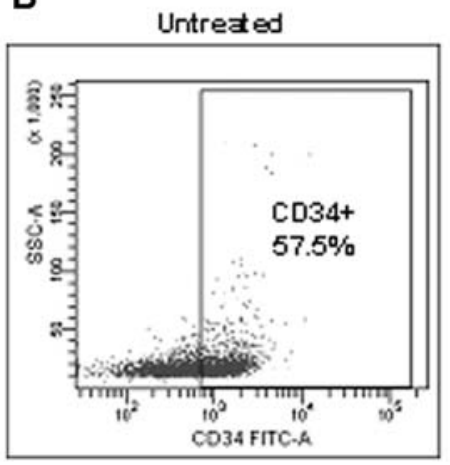

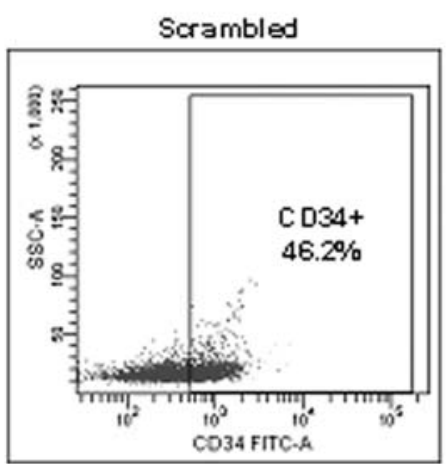

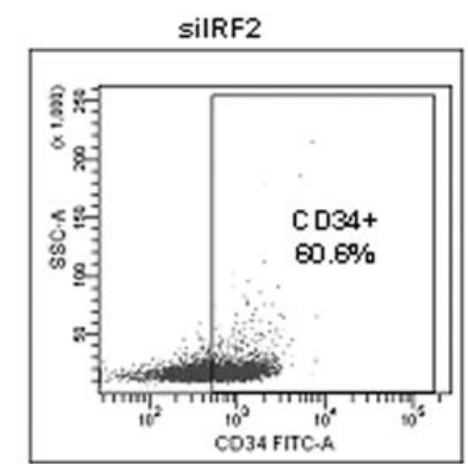

Figure 6. siIRF2 does not inhibit cell growth in normal haematopoietic CD34+ cells. (A) Cell growth analysis of untreated, scrambled siRNA and siIRF2-treated $\mathrm{CD}_{4}{ }^{+}$cells up to 7 days following treatment. siIRF2 treatment does not affect CD34+ cell growth. Some inhibition was observed in CD34 cells treated with scrambled siRNA. (B) CD34 ${ }^{+}$expression in untreated, scrambled siRNA and siIRF2-treated CD34 ${ }^{+}$cells 5 days following treatment was analysed by flow cytometry. Expression of CD34 was not affected by treatment of CD34+ cells with siIRF2. Some reduction was observed in cells treated with scrambled siIRF2.

\section{Discussion}

We demonstrated herein a novel siRNA test developed by us which uses a neomycin phosphotransferase gene linked to a target gene. Upon transcript degradation by siRNA, sensitivity to G418 was greatly increased. Thus, the reduction in cell numbers and cell viability, in the presence of G418, is representative of the level of target gene suppression. This test was confirmed by Western protein analysis at $48 \mathrm{~h}$ where a marked reduction of IRF2 protein level was seen in comparison to the control. This technique enables siRNA testing by a relatively simple, sensitive and rapid means (within $24 \mathrm{~h}$ ).

We next looked at the effect of IRF2 suppression in leukaemic TF-1 cells that express abnormally high levels of tumour suppressor IRF1. We hypothesized that high expression of IRF2, the antagonist of IRF1, acts to protect these cells from the tumour suppressor activity of IRF1, and thus, down-regulation of IRF2 must reduce this protection and result in the reduced cell growth. Indeed, leukaemic TF-1 cells transfected with siIRF2 showed marked downregulation of IRF2 at both mRNA and protein levels. Results from cell counts and colony assays revealed marked growth inhibition of TF-1 cells when treated with siIRF2. Remarkably, clonogenic suppression induced by targeting IRF2 results in relatively sustained growth inhibition: it is not just that the number of colony forming cells is reduced but also the proliferative activity of clonogenic cells that survived acute treatment with siIRF2 is significantly reduced (Fig. 3B, left panel).

Cell cycle analysis indicated that growth inhibition of siIRF2-treated cells was due to an accumulation of cells at $\mathrm{G}_{2} / \mathrm{M}$ at $48 \mathrm{~h}$. The latter correlates with the up-regulation of p21 $1^{\text {WAFI }}$ (Fig. 2E). Morphological analysis post-transfection also revealed a marked increase of cells in different phases of mitosis and abnormal polyploid cells. It was previously shown that normal cells that are arrested at $\mathrm{G}_{2} / \mathrm{M}$ do not synthesize DNA (16). We hypothesize that polyploid cell formation observed in leukaemic TF-1 cells results from the continued DNA synthesis occurring in $\mathrm{G}_{2} / \mathrm{M}$-arrested cells, the later apparently is due to the constitutive degradation of cdk inhibitor, p27 (17).

In light of the $\mathrm{G}_{2} / \mathrm{M}$ arrest observed, either differentiation or eventual cell death were expected in the siIRF2-treated cell population. In siIRF2-treated cells, evidence of myeloid differentiation was observed by flow cytometry and evidence of cell death by decreased cell counts following serum and IL-3 withdrawal. Collectively, our results show that IRF2 targeting alone appears to induce a number of features of myeloid differentiation such as growth arrest, $p 21^{\text {WAFI }}$ induction, reduced expression of primitive marker CD34, increased cellular granularity and cell enlargement. However, IRF2 
targeting alone does not appear to be sufficient to provide all signals that are needed for terminal differentiation of $\mathrm{TF}-1$ cells arrested in their differentiation during leukaemogenic transformation. It is relevant that TF-1 cells do not respond to the inhibitory effects of TGF- $\beta$ on cell-cycle progression that often induces cell differentiation (17).

Protein analysis revealed a steady increase of IRF1 upon treatment of TF-1 cells with siIRF2. This is of great importance as it may indicate an alteration of the IRF2 abrogation mechanism, whereby IRF2 also targets and inhibits IRF1 up-regulation. In addition, it highlights the specificity of siIRF2 treatment. siRNA mechanisms are sequence based and IRF1 and IRF2 share very high sequence homology. Thus, it is important that a sequence which affects IRF2 does not also affect IRF1. Since IRF1 functions as a tumour suppressor, down-regulation of this gene would prove undesirable. The specific targeting of IRF2 therefore indicates that the tumour suppressor effects including growth inhibition and cell cycle arrest were caused both by IRF2 suppression and IRF 1 up-regulation.

Targeting endogenous IRF2 in U937 cells (Neo-only cells) does not affect cell growth when cells were growing in media without G418. Our previous studies reported low levels of IRF2 and IRF1 expression in U937 cells (11). Upregulation of IRF1 expression in U937 cells by mutant $N$-ras results in growth suppression that can be abrogated by the enforced expression of IRF2 $(5,11)$. We hypothesise that IRF1 expression in U937 cells is not high enough to allow growth suppression when IRF2 levels are reduced in these cells by siIRF2. High expression of IRF1 appears to be a prerequisite for the efficient induction of tumour suppression in cancer cells by targeting IRF2. Similarly, targeting IRF2 in normal haematopoietic progenitor cells (CD34+ cells) did not induce growth suppression. It is relevant that the levels of IRF1 and IRF2, however, were similar in TF-1 cells and cord blood $\mathrm{CD} 4^{+}$cells stimulated with cytokines to support their viability and proliferation (data not shown). It is not clear why IRF2 down-regulation inhibits TF-1 leukaemic cells but does not affect normal haematopoietic progenitor cells. However, it should be noted that the IRF1:IRF2 ratio for $\mathrm{CD} 34^{+}$cells was $>2$-fold higher than in TF- 1 cells. In conclusion, our results show that suppression of IRF2 allows at least partial restoration of the growth arrest pathway, offering the potential for therapeutic treatment.

\section{Acknowledgements}

We would like to thank Leonie Gaudry for technical assistance with flow cytometry. This work was supported by a research project grant from The Cancer Council, New South Wales. The Children's Cancer Institute Australia for Medical Research is affiliated with the University of New South Wales, School of Women's and Children's Health and Sydney Children's Hospital.

\section{References}

1. Passegue E, Jamieson CHM, Ailles LE and Weissman IL: Normal and leukemic hematopoiesis: are leukemias a stem cell disorder or a reacquisition of stem cell characteristics? Proc Natl Acad Sci USA 100: 11842-11849, 2003.

2. Shih LY, Huang CF, Wang PN, Wu JH, Lin TL, Dunn P and Kuo MC: Acquisition of FLT3 or N-ras mutations is frequently associated with progression of myelodysplastic syndrome to acute myeloid leukemia. Leukemia 18: 466-475, 2004.

3. Appelbaum FR, Rowe JM, Radich J and Dick JE: Acute myeloid leukemia. Hematology 2001: 62-86, 2001.

4. MacKenzie KL, Dolnikov A, Millington M, Shounan Y and Symonds G: Mutant N-ras induces myeloproliferative disorders and apoptosis in bone marrow repopulated mice. Blood 93: 2043-2056, 1999

5. Shen SW, Dolnikov A, Passioura T, Millington M, Wotherspoon S, Rice A, MacKenzie KL and Symonds G: Mutant N-ras preferentially drives human CD34(+) hematopoietic progenitor cells into myeloid differentiation and proliferation both in vitro and in the NOD/SCID mouse. Exp Hematol 32: 852-860, 2004.

6. Passioura T, Dolnikov A, Shen S and Symonds G: N-rasinduced growth suppression of myeloid cells is mediated by IRF-1. Cancer Res 65: 797-804, 2005.

7. Abdollahi A, Lord KA, Hoffman-Liebermann B and Liebermann DA: Interferon regulatory factor-1 is a myeloid differentiation primary response gene induced by interleukin-6 and leukemia inhibitory factor: role in growth-inhibition. Cell Growth Differ 2: 401-407, 1991.

8. Green WB, Slovak ML, Chen IM, Pallavicini M, Hecht JL and Willman CL: Lack of IRF-1 expression in acute promyelocytic leukemia and in a subset of acute myeloid leukemias with del(5)(q31). Leukemia 13: 1960-1971, 1999.

9. Willman CL, Sever CE, Pallavicini MG, Harada H, Tanaka N, Slovak ML, Yamamoto H, Harada K, Meeker TC, List AF and Taniguchi T: Deletion of IRF-1, mapping to chromosome 5q31.1 in human leukemia and preleukemic myelodysplasia. Science 259: 968-971, 1993.

10. Guzman ML, Upchurch D, Grimes B, Howard DS, Rizzieri DA, Luger SM, Phillips GL and Jordan CT: Expression of tumorsuppressor genes interferon regulatory factor 1 and deathassociated protein kinase in primitive acute myelogenous leukemia cells. Blood 97: 2177-2179, 2001.

11. Passioura T, Shen S, Symonds G and Dolnikov A: A retroviral library genetic screen identifies IRF-2 as an inhibitor of N-rasinduced growth suppression in leukemic cells. Oncogene 24: 7327-7336, 2005.

12. Oshima S, Nakamura T, Namiki S, Okada E, Tsuchiya K, Okamoto R, Yamazaki M, Yokota T, Aida M, Yamaguchi Y, Kanai $\mathrm{T}$, Handa $\mathrm{H}$ and Watanabe $\mathrm{M}$ : Interferon regulatory factor 1 (IRF-1) and IRF-2 distinctively up-regulate gene expression and production of interleukin-7 in human intestinal epithelial cells. Mol Cell Biol 24: 6298-6310, 2004.

13. Perambakam S, Li B and Preisler H: Quantitation of interferon regulatory factor transcripts in patients with acute myeloid leukemia. Cancer Invest 19: 346-351, 2001.

14. Sledz CA, Holko M, De Veer MJ, Silverman RH and Williams BRG: Activation of the interferon system by shortinterfering RNAs. Nat Cell Biol 5: 834-839, 2003.

15. Grobe $\mathrm{K}$ and Powell LD: Role of protein kinase $\mathrm{C}$ in the phosphorylation of CD33 (Siglec-3) and its effect on lectin activity. Blood 99: 3188-3196, 2002.

16. Woo RA and Poon RYC: Activated oncogenes promote and cooperate with chromosomal instability for neoplastic transformation. Genes Dev 18: 1317-1330, 2004.

17. Schepers H, Wierenga ATJ, Eggen BJL and Vellenga E: Oncogenic Ras blocks transforming growth factor-beta-induced cellcycle arrest by degradation of p27 through a MEK/Erk/SKP2dependent pathway. Exp Hematol 33: 747-757, 2005. 